\section{Live mammalian cell arrays}

\author{
Kristina Woodruff ${ }^{1,2}$, Luis M Fidalgo ${ }^{1,2}$, \\ Samy Gobaa ${ }^{1}$, Matthias P Lutolf ${ }^{1} \&$ Sebastian J Maerkl ${ }^{1}$
}

\begin{abstract}
High-content assays have the potential to drastically increase throughput in cell biology and drug discovery, but handling and culturing large libraries of cells such as primary tumor or cancer cell lines requires expensive, dedicated robotic equipment. We developed a simple yet powerful method that uses contact spotting to generate high-density nanowell arrays of live mammalian cells for the culture and interrogation of cell libraries.
\end{abstract}

Cell-based assays and the tools used to perform them are constantly undergoing improvements toward higher experimental throughput, reduced reagent consumption and advanced control of the cell microenvironment ${ }^{1,2}$. There are currently two main approaches for conducting high-content cell-culture experiments. In the first, cells are cultured in microtiter plates, and robotic equipment is used to perform all necessary fluidic operations ${ }^{3}$. Although effective, this method remains unavailable to researchers in many laboratories because of the requirement for expensive robotics. A second approach is reverse transfection ${ }^{4-6}$, but complex cell libraries cannot be investigated by this method.

As an alternative, contact spotting offers an affordable yet highthroughput platform. This technique is extensively used to generate high-density DNA and protein arrays, and has been adapted to other purposes ${ }^{7}$. To date, contact spotting has not been used to array live mammalian cells because of rapid spot evaporation and consequent cell death. Therefore, arraying of live mammalian cells has been limited to inkjet printing, which lacks the ability to handle a large number of different samples ${ }^{8,9}$.

Large collections of mammalian cell lines have recently become available, including primary tumor and cancer cell lines ${ }^{10}$, stably transfected expression cell lines and GFP-fusion libraries ${ }^{11}$. Novel approaches are required to efficiently assemble complex arrays of hundreds to thousands of genetically diverse cells. We developed a simple, fast and scalable method that uses standard microarray printing tools to generate high-density nanowell arrays. A minimal sample requirement of 500 cells enables the interrogation of cells that are available in limited quantities or cells that cannot be expanded because of undesirable changes in phenotype or genotype, as in the case of stem cells and cancer cell lines ${ }^{12}$.

We generated live mammalian cell arrays (LMCAs) of both primary cells and commonly used cell lines. We collected cells from standard culture formats and resuspended them in Percoll, a high-density, cell-compatible colloidal suspension routinely used for the isolation of primary cells. Cells plated in conical 384-well plates were taken up by a microarray spotting pin and delivered to a 48-well poly(dimethylsiloxane) (PDMS) array $\left(\sim 20\right.$ wells $\left.\mathrm{cm}^{-2}\right)$ or a 675 -well acrylic array $\left(\sim 36\right.$ wells $\left.\mathrm{cm}^{-2}\right)$ adhered to a coverslip or glass slide, respectively (Supplementary Fig. 1). For comparison, the density of a 1,536-well plate is 14 wells $\mathrm{cm}^{-2}$. Each nanowell had 500-nl capacity and was filled with medium before array spotting to ensure viability of the added cells and to enable the automated handling of nanoliter volumes of live cells. Once arrayed, cells were incubated in a high-humidity environment for $60 \mathrm{~min}$ to allow them to attach to the glass surface (Supplementary Fig. 1). After attachment, the array was submerged in medium for culturing and imaging (Fig. 1a).

Arraying cells suspended in standard medium resulted in poor performance in terms of cell transfer, requiring hundreds of thousands of cells per sample (Fig. 1b). Resuspending cells in Percoll improved the efficiency of transfer by several orders of magnitude. This approach allowed spots containing $\sim 40$ cells to be reproducibly arrayed from samples of 1,000 cells (Fig. 1b). The number of cells delivered to each nanowell can be tuned by the initial sample cell density, so that $\sim 10-400$ cells can be delivered from input samples of 500-50,000 cells (Fig. 1b). The number of cells transferred decreased with successive printings, but it was nonetheless possible to print repeatedly from the same sample (Supplementary Fig. 2).

Cross-contamination between nanowells can, in principle, occur by two mechanisms: (i) carryover of cells between spots by the pin and (ii) direct transfer of cells between nanowells when medium is added to the nanowell array. To investigate contamination via carryover, we alternated spotting samples of cells and, as negative controls, samples of medium, with a pin-wash cycle between each sample (Online Methods). We found no cells in the negative control nanowells (Fig. 1c). To investigate contamination during addition of medium, we spotted an array of cells in which most wells were left empty. These originally empty wells remained uncontaminated after the exchange of medium (Fig. 1d). When printing larger arrays in which all nanowells were programmed with cells, we identified a low degree of contamination, amounting to 2 of 48 nanowells (4\%; Supplementary Fig. 3).

To assess the viability of cells after the arraying process, we prepared 48 separate aliquots of NIH-3T3 fibroblasts on a source plate and transferred each aliquot to a separate nanowell. We washed the pin after spotting each sample, thus simulating the arraying of 48 different cell samples (Fig. 1e). The fibroblasts stably expressed a red fluorescent protein that rapidly disperses upon cell lysis, allowing fluorescence to serve as a direct measure of cell viability. After the cells were arrayed, they remained viable and quickly became

${ }^{1}$ Institute of Bioengineering, School of Engineering and School of Life Science, École Polytechnique Fédérale de Lausanne, Switzerland. ${ }^{2}$ These authors contributed equally to this work. Correspondence should be addressed to S.J.M. (sebastian.maerkl@epfl.ch). 
Figure 1 A method to array live mammalian cells. (a) Schematic of the method. (b) Quantification of cell transfer. The number of cells transferred to a nanowell (cells spotted) from source wells containing the indicated number of cells (cells plated) with and without Percoll. Error bars, s.d. $(n=3)$. (c) Micrograph of NIH-3T3 fibroblasts expressing tdTomato spotted in the top row, to assess cell carryover by the spotting pin.

(d) Micrograph of an array in which three wells were spotted with $3 \mathrm{~T} 3$ fibroblasts expressing tdTomato (first row) and three wells were spotted with HEK293 cells expressing GFP (third row) to assess direct cell transfer across wells.

(e) Micrograph of a 48-well array seeded in all wells with NIH-3T3 fibroblasts expressing tdTomato and imaged $2 \mathrm{~d}$ after spotting.

confluent in the nanowell (Supplementary Fig. 4). We also tested the viability of cells in LMCAs after freezing them. We could freeze and thaw both the 48-well PDMS and the 675-well acrylic LMCAs without substantial effects on cell viability (Supplementary Fig. 5).

The LMCA method can be applied to a variety of mammalian cell types, including NIH-3T3 fibroblasts, human liver carcinoma (Hep G2), human embryonic kidney (HEK) and Chinese hamster ovary (CHO) cells (Fig. 2a and Supplementary Fig. 6). We also applied this method to primary cells. We spotted human bone marrow-derived mesenchymal stem cells (hMSCs) and maintained them in culture for over 2 weeks with no contamination or detectable decrease in cell viability (Fig. 2a).

To test the scalability of this technique, we also used acrylic arrays with 675 nanowells (Fig. 2b). The 675-nanowell arrays are commercially available (ALine, Inc.), and dimensions such as well

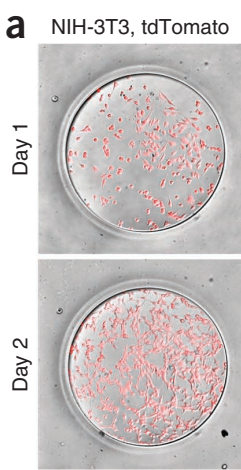

b

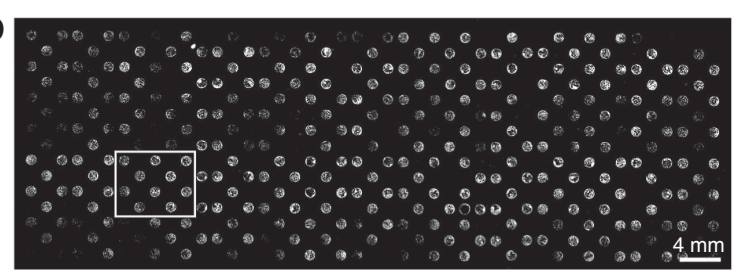

C

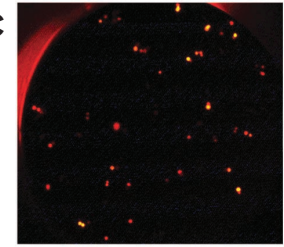

Hep G2
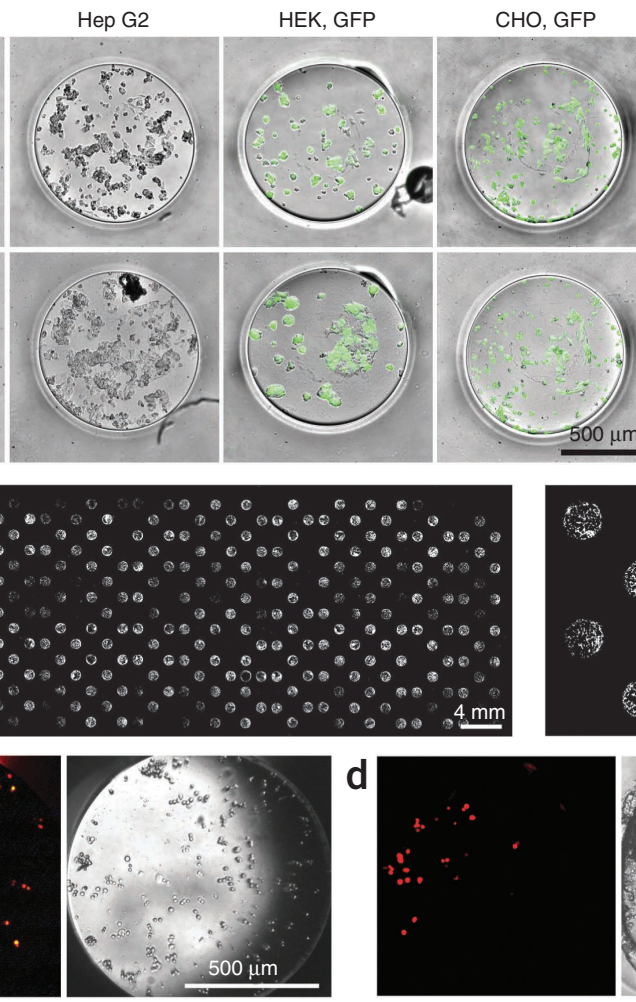

b
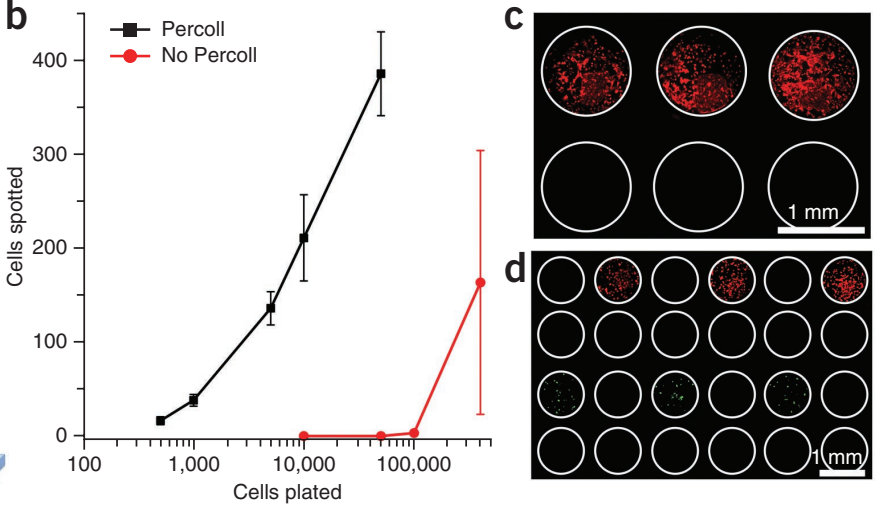

e

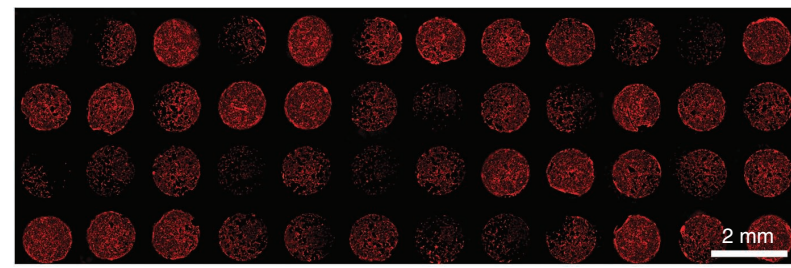

size and pitch can be customized. Our array had wells of $1 \mathrm{~mm}$ diameter and $1.5 \mathrm{~mm}$ pitch, enabling the entire substrate to fit on a microscope slide. A single 4-well microtiter plate could thus be used to culture up to 2,700 cell types. We programmed 339 of the 675 wells with cells (Fig. 2b). Twenty-four hours after spotting, 331 of the wells contained viable cells, indicating a $2 \%$ failure rate in cell transfer. Forty wells that were not originally spotted contained cells ( 32 contained a single cell and the rest contained two or three cells). Therefore, $7 \%$ (48 of 675) of the nanowells were either contaminated or contained no cells when they should have had cells.

LMCAs are compatible with transfection. The standard reversetransfection protocol uses microarraying pins that generate smalldiameter spots $(120-150 \mu \mathrm{m})$ that dry rapidly and uniformly ${ }^{4}$. However, for our LMCA nanowells, spots of $1 \mathrm{~mm}$ diameter were required. Larger spots tend to dry slowly and unevenly, causing the majority of the transfection reagent to concentrate at the edges of the nanowell. Reverse transfection has been achieved using the large wells of 24-well

Figure 2 | Applications of the arraying method. (a) Brightfield and fluorescence images of five cell types spotted and cultured in PDMS nanowell arrays. hMSCs were cultured for up to $14 \mathrm{~d}$ (insets show magnification of boxed regions); all other cell types were cultured for up to $2 \mathrm{~d}$. (b) Fluorescence micrograph of an entire 675well acrylic array in which 339 of the wells were spotted with 3T3 fibroblasts by simultaneously using three spotting pins. Boxed region of the array is magnified on the right. (c) Fluorescence (left) and brightfield (right) images, $20 \mathrm{~h}$ after reverse transfection of $\mathrm{CHO}$ cells with plasmid encoding tdTomato (spotting of a lipid-DNA mixture) in 48 nanowells of a PDMS array followed by manual addition of cells. (d) Regular transfection of $\mathrm{CHO}$ cells spotted into the 675well array, followed by spotting of tdTomato plasmid transfection mixture into the wells. Images were acquired $45 \mathrm{~h}$ after transfection. 


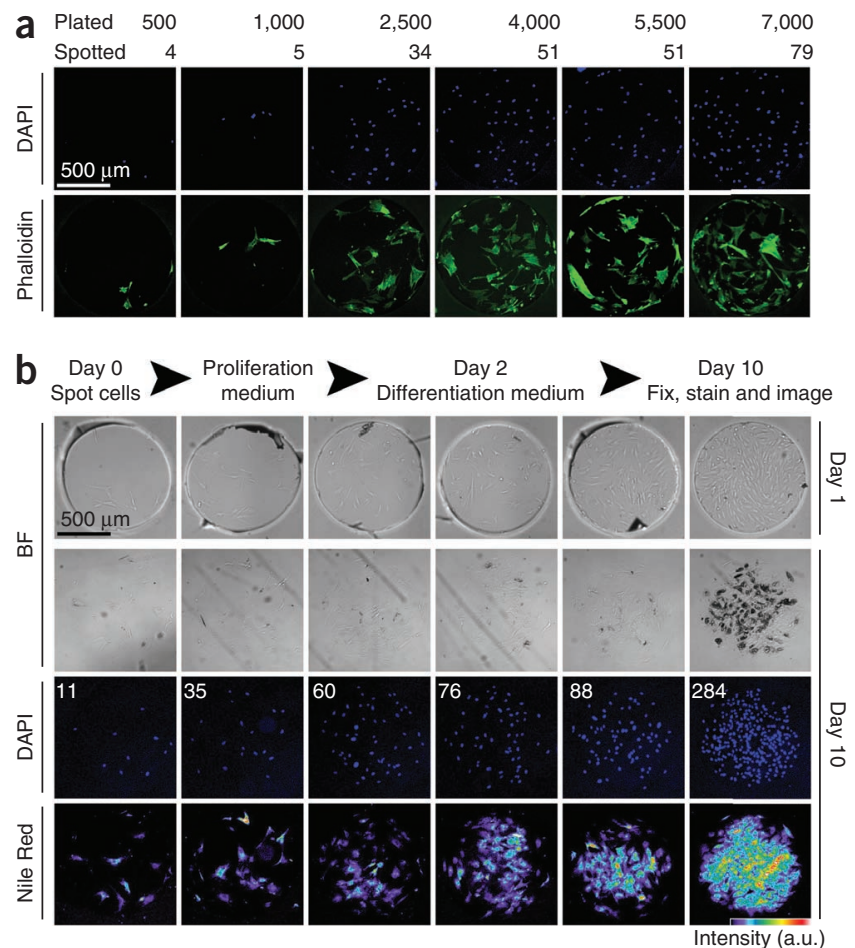

Figure 3 | Human mesenchymal stem cell array. (a) DAPI and phalloidin stains of hMSCs spotted into the 48-well PDMS array from sample wells containing 500-7,000 cells. (b) hMSC differentiation scheme and micrographs of individual nanowells from the PDMS array. Brightfield (BF), DAPI and Nile Red stains are shown. On the DAPI micrographs, total cell numbers are indicated. In the Nile Red pseudocolor scale, the highest adipogenic differentiation is indicated by red and white pixels.

microtiter plates $^{13}$, but the microliter volumes of reagents administered to each well preclude the use of a microarrayer. We developed a method that uses the microarraying pin for automated deposition of homogeneous lipid-DNA spots into the nanowells of our PDMS arrays. Upon introducing cells into the wells with a micropipette, we achieved $32 \%$ transfection efficiency, with transfected cells uniformly distributed throughout the nanowell (Fig. 2c). Manually depositing larger volumes of reagent did not improve efficiency (Supplementary Fig. 7), suggesting that our spotting protocol was sufficient to increase the local concentration of the transfection reagent at the site of cell attachment. Our method addresses two limitations of standard reverse transfection: the physical separation of cells on our array prevents cross-contamination and also allows for the inclusion of additional soluble factors.

We also tested a regular transfection approach, spotting cells before adding lipid-DNA complexes. Using the acrylic 675-well array, transfection efficiency was $20 \%$ for the wells with manually added transfection reagent (Fig. 2d). Efficiency was lower for wells in which the lipid-DNA was introduced by a microarraying pin (13\%), most likely due to the limited $\sim 20 \mathrm{nl}$ transfer volume of the pin (Supplementary Fig. 7). We achieved considerably lower efficiency with the PDMS 48-well array (Supplementary Fig. 7).

We also spotted hMSCs at different densities in a controlled fashion, which resulted in a cell-transfer efficiency similar to that observed with standard cell lines (Fig. 3a). We could spot $\sim 5$ cells into the nanowells when using a sample well containing as few as 500 cells, and up to $\sim 80$ cells when using samples of 7,000 cells (Supplementary Fig. 8).
We repeatedly used each sample well to spot 4 nanowells, which resulted in fewer cells deposited into each subsequent nanowell.

We investigated the adipogenic differentiation potential of hMSCs cultured on arrays for up to $10 \mathrm{~d}$. We spotted the hMSCs at a variety of densities and incubated the array in proliferation medium for $2 \mathrm{~d}$. Then we placed the array into differentiation medium for $8 \mathrm{~d}$. At the end of the 10-d period, we fixed the array, stained it with DAPI and Nile Red, and imaged it. Wells that initially contained larger numbers of cells exhibited pronounced adipogenic differentiation, as measured by Nile Red staining (Fig. 3b). These cells produced large lipid vesicles that appeared as dark spots in brightfield. In contrast, wells seeded at lower densities contained cells that differentiated to a lesser extent and produced fewer and smaller lipid vesicles.

In summary, we generated high-density LMCAs with a variety of mammalian cell types, using contact spotting. This method requires only a standard contact microarrayer and a nanowell array that can be easily fabricated ${ }^{14}$ or commercially acquired (ALine, Inc.). The cell arrays could also be used in conjunction with arrays of hydrogels and biomolecules or for screening of artificial extracellular matrix ${ }^{15,16}$. The high-throughput approach to cell handling will enable the parallel culture and analysis of large libraries of mammalian cells.

\section{METHODS}

Methods and any associated references are available in the online version of the paper.

Note: Supplementary information is available in the online version of the paper.

\section{ACKNOWLEDGMENTS}

We thank D. Hacker (Laboratory of Cellular Biotechnology, Institute of Bioengineering, École Polytechnique Fédérale de Lausanne) for kindly providing GFP-expressing CHO and HEK cells, and V.M. Mangoua, A. Ranga and A. Negro for help with the project. The work was funded by a Marie Curie fellowship (FP7-PEOPLE-2009-IEF 252457), by a SystemsX.ch Research, Technology and Development grant: DynamiX (2008/005), a Swiss National Science Foundation Pro-Doc grant (PDFMP3 137065), a European Young Investigator grant PE002-117115/1 to M.P.L. and the École Polytechnique Fédérale de Lausanne.

\section{AUTHOR CONTRIBUTIONS}

L.M.F., K.W., M.P.L. and S.J.M. designed experiments and developed the method. L.M.F. and K.W. performed experiments. S.G. assisted with cell culturing and image processing and provided transfection reagents. L.M.F., K.W., M.P.L. and S.J.M. wrote the paper. S.J.M. conceived the idea.

\section{COMPETING FINANCIAL INTERESTS}

The authors declare no competing financial interests.

Reprints and permissions information is available online at http://www.nature. com/reprints/index.html.

1. Yarmush, M.L. \& King, K.R. Annu. Rev. Biomed. Eng. 11, 235-257 (2009).

2. Xu, F.F. et al. Biofabrication 3, 034101 (2011).

3. Taylor, D.L. Methods Mol. Biol. 356, 3-18 (2006).

4. Ziauddin, J. \& Sabatini, D.M. Nature 411, 107-110 (2001).

5. Wheeler, D.B., Carpenter, A.E. \& Sabatini, D.M. Nat. Genet. 37, S25-S30 (2005).

6. Bailey, S.N., Sabatini, D.M. \& Stockwell, B.R. Proc. Natl. Acad. Sci. USA 101, 16144-16149 (2004).

7. Hart, T., Zhao, A., Garg, A., Bolusani, S. \& Marcotte, E.M. PLoS ONE 4 e7088 (2009).

8. Lee, M.Y. et al. Proc. Natl. Acad. Sci. USA 105, 59-63 (2008).

9. Yusof, A. et al. Lab Chip 11, $2447-2454$ (2011).

10. Neve, R.M. et al. Cancer Cell 10, 515-527 (2006).

11. Cohen, A.A. et al. Science 322, 1511-1516 (2008).

12. Lutolf, M.P., Gilbert, P.M. \& Blau, H.M. Nature 462, 433-441 (2009).

13. Fujita, S.S. et al. J. Biosci. Bioeng. 104, 5 (2007).

14. Jackman, R.J.R., Duffy, D.C.D., Ostuni, E.E., Willmore, N.D.N. \& Whitesides, G.M.G. Anal. Chem. 70, 2280-2287 (1998).

15. Mei, Y. et al. Nat. Mater. 9, 768-778 (2010).

16. Gobaa, S. et al. Nat. Methods 8, 949-955 (2011). 


\section{ONLINE METHODS}

Substrate fabrication. PDMS membranes were molded from SU8 structures fabricated on silicon wafers using standard photolithography methods ${ }^{17}$. Two layers of approximately $200 \mu \mathrm{m}$ thickness were spun and baked before exposure and development. Before use, the structures were silanized using $1 \mathrm{H}, 1 \mathrm{H}, 2 \mathrm{H}, 2 \mathrm{H}-$ perfluorooctyl trichloro silane (Sigma) as previously described ${ }^{18}$. The wafers were spin coated with PDMS (RTV615, General electric, $60 \mathrm{~s}$ at 150 r.p.m. and $120 \mathrm{~s}$ at 350 r.p.m.). The coated wafer was degassed for $5 \mathrm{~min}$ and left to reflow for $25 \mathrm{~min}$ at room temperature before baking for $30 \mathrm{~min}$ at $80{ }^{\circ} \mathrm{C}$. This process resulted in a perforated membrane of $\sim 200 \mu \mathrm{m}$ thickness. The membrane had 48 wells of $1 \mathrm{~mm}$ of diameter with a pitch of $2.25 \mathrm{~mm}$.

The patterned PDMS was cut from the wafer and transferred to glass microscope coverslips. Separately, an array of BSA that matched the pitch of the membrane was spotted onto an epoxycovered microscope slide. The membrane wells on the coverslip were manually aligned to the BSA spots on the slide using a stereoscope, providing a simple method to ensure alignment between the arraying robot and the microfabricated substrate. For some samples, a PDMS frame surrounding the membrane was manually cut and bonded to the coverslip using oxygen plasma. This frame provided an efficient medium container around the membrane and enabled high-resolution imaging of the coverslip-supported cell array. Samples were UV light-sterilized after assembly and coated with fibronectin (Sigma) either by spotting a concentrated suspension $(1 \mathrm{mg} / \mathrm{ml})$ or by 1 -h incubation in a dilute solution $(50 \mu \mathrm{g} / \mathrm{ml})$.

The 675-well array plastic substrates were purchased from ALine, Inc. The arrays consisted of a $500 \mu \mathrm{m}$ acrylic layer and a $50 \mu \mathrm{m}$ silicone adhesive layer. They had wells of $1 \mathrm{~mm}$ diameter with $1.5 \mathrm{~mm}$ pitch and were adhered to glass microscope slides by passing them slowly through lamination rollers at $60^{\circ} \mathrm{C}$. Arrays were not treated with fibronectin before cell spotting.

Cell culture. Cells were cultured in Dulbecco's modified Eagle medium (DMEM) supplemented with 10\% FBS and 1\% penicillinstreptomycin at $37^{\circ} \mathrm{C}$ and $5 \% \mathrm{CO}_{2}$. For long-term assays, medium supplemented with antibiotic-antimycotic was used. Cells were passaged every $2 \mathrm{~d}$ using TrypleE express or trypsin. All culture reagents were acquired from Gibco (Life Technologies). NIH-3T3 fibroblasts stably expressed GFP or tdTomato fluorescent protein, whereas CHO and HEK293 cells expressed GFP (100\% and 50\% of the cells expressing fluorescent protein, respectively). Other cell lines used included Hep G2 cells, human MSCs and wildtype $\mathrm{CHO}$ cells.

Cell spotting. Cells were collected from T75 or T25 flasks and washed. After cells were adjusted to the desired concentration, they were transferred to $500 \mu$ l Eppendorf tubes in $20-\mu$ l aliquots. These aliquots were centrifuged at $300 \mathrm{~g}$ for $3 \mathrm{~min}$ and resuspended in a Percoll standard solution (700 $\mu$ l Percoll (Sigma), $100 \mu \mathrm{l}$ $10 \times$ PBS (Gibco) and $200 \mu \mathrm{l}$ Milli-Q water). For longer spotting programs, $10 \times$ PBS was replaced with $10 \times$ DMEM. Cells resuspended in Percoll were plated in 5- $\mu$ l aliquots onto conical-well, poly(propylene) 384-well plates (Arrayit). Cells were spotted using a pin with a $100-\mu \mathrm{m}$-wide channel, a $1.25 \mu$ l uptake volume and a nominal $1.5 \mathrm{nl}$ transfer volume (WCMP, Arrayit). Spotting parameters were $2 \times 1 \mathrm{~s}$ inking time, $2 \times 1 \mathrm{~s}$ print time per well.
The washing protocol consisted of four alternating washes with ethanol and water. The first three washes were $2 \mathrm{~s}$ each, and the last wash was $5 \mathrm{~s}$, with $5 \mathrm{~s}$ of drying after the last wash. Including spotting and washing, the total time per sample was approximately $30 \mathrm{~s}$, and therefore an array of 48 different samples was completed in under $25 \mathrm{~min}$. Before spotting, the 48-well PDMS arrays were prefilled by manual pipetting $\sim 0.5 \mu$ l of $0.7 \times$ culture medium into each well. For the 675 -well arrays, wells were prefilled by submerging the entire array in $0.7 \times$ medium. Air bubbles were removed from wells by pipetting. The array was then slowly removed from the medium, and excess liquid was wiped from the surface. The same printing conditions used for the 48-well array were used for the 675-well array, but only with one $1.5 \mathrm{~s}$ water wash and $3 \mathrm{~s}$ of drying. During spotting, the humidity in the chamber was set to $70 \%$ to reduce evaporation of medium from the wells without causing the printing malfunction that is observed at higher humidity.

Array culture. Immediately after spotting, substrates were placed in a petri dish that contained warm Milli-Q water to prevent evaporation during transport to the incubator and during cell attachment. To preserve the integrity of the array, the samples were placed on a PDMS block adhered at the center of the dish, ensuring that the water contained in the dish did not come in contact with the sample. After incubation at $37^{\circ} \mathrm{C}(1 \mathrm{~h}$ for the 48-well array and $3 \mathrm{~h}$ for the 675-well array) the cell arrays were covered in medium for culture. To this end, either the coverslip was detached from the epoxy slide and placed in a different petri dish or, for samples with a PDMS frame, medium was directly added to the array.

To prepare arrays for freezing, LMCAs were placed in small plastic containers containing 90\% FBS and 10\% DMSO. The container was then placed into a cryobox with isopropanol, and frozen at $-80^{\circ} \mathrm{C}$ overnight. The arrays were thawed by submersion in a beaker of PBS contained in a $37^{\circ} \mathrm{C}$ water bath. Once thawed, arrays were washed three times in culture medium to remove residual DMSO.

For primary cell arrays, hMSCs were spotted onto a 48-well PDMS array following the procedure used for other cell types. hMSC proliferation medium consisted of $\alpha$-minimum essential medium (Invitrogen), 10\% FBS (Hyclone), $1 \mathrm{ng} / \mathrm{ml}$ fibroblast growth factor 2 (FGF2; R\&D Systems), $2 \mathrm{mM}$ L-glutamine (Sigma-Aldrich) and 1\% penicillin-streptomycin (Invitrogen). Adipogenic differentiation medium contained low-glucose DMEM (Invitrogen), 20\% FCS (Hyclone), 0.5 mM 3-isobutyl-1methylxanthine (IBMX; Sigma-Aldrich), $60 \mu \mathrm{M}$ indomethacin (Fluka) and $1 \mu \mathrm{M}$ dexmethasone (Sigma-Aldrich). The arrays were fixed in $4 \%$ paraformaldehyde for $15 \mathrm{~min}$ at room temperature. The PDMS membrane was removed before cell staining. Nuclei were stained with a $10 \mu \mathrm{g} / \mathrm{ml}$ solution of DAPI (SigmaAldrich) in PBS. Lipid vesicles were stained with a $1 \mu \mathrm{g} / \mathrm{ml}$ solution of Nile Red (Sigma-Aldrich) in PBS. The cell cytoskeleton was visualized by staining with phalloidin-Alexa Fluor 488 (Invitrogen) according to the manufacturer's instructions.

Transfection. For the reverse-transfection approach, glass slides coated with poly(L-lysine) (Electron Microscopy Sciences) were used. We diluted $0.37 \mu \mathrm{l}$ DNA (at $1.8 \mu \mathrm{g} / \mu \mathrm{l}$ ) into $11.13 \mu \mathrm{l}$ of a dextran solution. We added $2 \mu$ l Fugene (Roche), and the mixture 
was incubated for $15 \mathrm{~min}$. Then, $46 \mu \mathrm{l}$ polyvinyl alcohol (PVA) was added, and the mixture was spotted into the nanowells using a pin with a $500-\mu \mathrm{m}$-wide channel, a $1.25 \mu \mathrm{l}$ uptake volume and a 15-25 nl transfer volume (WCMPL, Arrayit). Arrays were allowed to dry, after which cells were introduced into the nanowells by manual pipetting. Spotting conditions for the lipid-DNA mixture were identical to those used for cells except that only one water wash (1 s) with $3 \mathrm{~s}$ drying was used between samples, and three stamps per well were implemented. Dextran $\left(\mathrm{M}_{\mathrm{r}} \sim 40,000\right.$, Sigma) was prepared at $45 \mathrm{mg} / \mathrm{ml}$ and PVA (molecular weight, 25,000 ; Polysciences) was prepared at $0.5 \%(\mathrm{v} / \mathrm{v})$ as described ${ }^{13}$. For regular transfection, cells were spotted as previously described. Two hours after spotting, a lipid-DNA mixture was prepared and spotted into the nanowells as described above, with the modification that fibronectin $(1 \mathrm{mg} / \mathrm{ml})$ was used instead of PVA. Regular transfection arrays were not submerged in medium until $20 \mathrm{~h}$ after initial cell spotting.

Array imaging. Imaging of the 48-nanowell array was performed on a Nikon Ti automated microscope using custom-written software to determine the positions to image trigonometrically and acquire the multichannel images. Images were typically acquired using $10 \times$ magnification to capture an entire well in the field of view. Array images were collaged using ImageJ. Image J was also used for cell counting. First, images where thresholded and inverted. Then, single cells were counted using the particleanalysis functions. Cells that were aggregated or too close for direct counting were counted by measuring their area and calculating the number using a proportion obtained from manual counting of a group of cells from the same sample. DAPI-stained hMSCs were counted using the count nuclei program of Metamorph.

Imaging of the 675-nanowell array was performed on a Nikon TI automated microscope using NIS Elements. Each well was imaged with a $4 \times$ objective in brightfield and fluorescence mode. The resulting 675 images were first brightness-adjusted and converted to jpeg format from tiff format, and then stitched together using the Grid/Collection Stitching plugin in Fiji ${ }^{19}$.

17. Xia, Y. \& Whitesides, G. Annu. Rev. Mater. Sci. 28, 153-184 (1998).

18. Ferguson, G.S., Chaudhury, M.K., Biebuyck, H.A. \& Whitesides, G.M. Macromolecules 26, 5870-5875 (1993).

19. Preibisch, S., Saalfeld, S. \& Tomancak, P. Bioinformatics 25, 1463-1465 (2009). 